Current version: 24 October 2018

\title{
de Sitter spacetime: effects of metric perturbations on geodesic motion
}

\author{
Donato Bini* $\S^{\dagger}$, Giampiero Esposito and Andrea \\ Geralico $^{\S \ddagger}$ \\ * Istituto per le Applicazioni del Calcolo "M. Picone," CNR, 00185 Rome, Italy \\ $\S$ ICRA, University of Rome "La Sapienza," 00185 Rome, Italy \\ $\dagger$ INFN, Sezione di Firenze, 00185 Sesto Fiorentino (FI), Italy \\ ฯ INFN, Sezione di Napoli, Complesso Universitario di Monte S. Angelo, Via \\ Cintia, Edificio 6, 80126 Napoli, Italy \\ $\ddagger$ Physics Department, University of Rome "La Sapienza," 00185 Rome, Italy
}

\begin{abstract}
Gravitational perturbations of the de Sitter spacetime are investigated using the Regge-Wheeler formalism. The set of perturbation equations is reduced to a single second order differential equation of the Heuntype for both electric and magnetic multipoles. The solution so obtained is used to study the deviation from an initially radial geodesic due to the perturbation. The spectral properties of the perturbed metric are also analyzed. Finally, gaugeand tetrad-invariant first-order massless perturbations of any spin are explored following the approach of Teukolsky. The existence of closed-form, i.e. Liouvillian, solutions to the radial part of the Teukolsky master equation is discussed.

PACS number: 04.20.Cv, 04.30.Nk
\end{abstract}

\section{Introduction}

de Sitter spacetime is extensively investigated in the literature from different points of view. In fact, it is particularly relevant for inflation 1] and hence the physics of the very early universe. Furthermore, there are many recent experimental evidences and astronomical observations which indicate that the cosmological constant in the universe is positive leading to an accelerating expansion (see, e.g., Ref. [2] and references therein). The pioneering work of Gibbons and Hawking [3] extending to cosmological horizons the concepts of thermodinamics and particle creation previously introduced for black hole event horizons opened the way to the study of the properties of quantum field theory on de Sitter space, especially the temperature and entropy (see, e.g., Refs. 4, 5, 6, 7]), and on asymptotically de Sitter spaces containing black holes. In this respect, it proved useful to express the de Sitter metric in static form (see, e.g., Ref. [8]).

We are interested here in studying metric perturbations in the static region of the de Sitter spacetime between the origin and the cosmological horizon. We will then use the Regge-Wheeler 9] formalism to decompose the perturbations in tensor harmonics and Fourier transform it with respect to time, as customary. We will account for perturbations of both parity, and investigate the features of geodesic motion in the perturbed gravitational field. In particular, we will consider an initially radial geodesic 
in the unperturbed spacetime and study how the motion deviates from maintaining purely radial as a result of the perturbation. Furthermore, we will also consider gauge- and tetrad-invariant first-order massless perturbations of any spin following the approach of Teukolsky [10, and discuss the existence of closed-form, i.e. Liouvillian, solutions to the radial equation. Note that our results are consistent with those of Refs. [11] and [12] concerning the perturbations of the Schwarzschild-de Sitter spacetime, which however focused on the stability of the de Sitter spacetime in the presence of the black hole and on the scattering properties of both the event and cosmological horizons.

Section 2 summarizes what is known about radial geodesics in de Sitter spacetime, while metric perturbations for both electric and magnetic multipoles are discussed in section 3. The equations for perturbations to radial geodesics are integrated in section 4 , while section 5 contains the derivation of Liouvillian solutions to the radial part of the Teukolsky master equation for massless perturbations of any spin. Concluding remarks are presented in section 6 , while relevant details are given in the appendices.

\section{Radial geodesics in de Sitter spacetime}

Consider de Sitter spacetime, whose line element written in spherical-like coordinates is given by

$$
\mathrm{d} s^{2}=N^{2} \mathrm{~d} t^{2}-N^{-2} \mathrm{~d} r^{2}-r^{2}\left(\mathrm{~d} \theta^{2}+\sin ^{2} \theta \mathrm{d} \phi^{2}\right),
$$

where $N=\left(1-H^{2} r^{2}\right)^{1 / 2}$ denotes the "lapse" function. The spacetime region which can be accessed is the ball of radius $1 / H$ and center at the origin. Timelike radial geodesics are characterized by their 4 -velocity

$$
U=\Gamma\left(\partial_{t}+\zeta \partial_{r}\right)
$$

with

$$
\Gamma=\frac{\mathrm{d} t}{\mathrm{~d} \tau}=\frac{E}{N^{2}}, \quad \frac{\mathrm{d} r}{\mathrm{~d} \tau}=\Gamma \zeta= \pm \sqrt{E^{2}-N^{2}},
$$

so that $\tau$ denotes the proper time and

$$
\zeta= \pm \frac{N^{2}}{E} \sqrt{E^{2}-N^{2}}
$$

$E$ is the Killing constant associated with the stationarity of the metric. It is convenient to introduce an orthonormal frame adapted to the static observer family (at rest with respect to the coordinates) with basis vector fields

$$
e_{\hat{0}}=N^{-1} \partial_{t}, \quad e_{\hat{r}}=N \partial_{r}, \quad e_{\hat{\theta}}=1 / r \partial_{\theta}, \quad e_{\hat{\phi}}=1 /(r \sin \theta) \partial_{\phi},
$$

with dual basis of 1 -forms

$$
\omega^{\hat{0}}=N d t, \quad \omega^{\hat{r}}=-N^{-1} d r, \quad \omega^{\hat{\theta}}=-r d \theta, \quad \omega^{\hat{\phi}}=-r \sin \theta d \phi .
$$

With respect to this frame the geodesic 4-velocity vector field can be written as

$$
U=\gamma\left(e_{\hat{0}}+\nu e_{\hat{r}}\right)
$$

where

$$
\gamma=\Gamma N, \quad \nu=N^{-2} \zeta
$$

The parametric equations of the geodesics corresponding to initial values

$$
t(0)=0, \quad r(0)=0, \quad \theta(0)=0, \quad \phi(0)=0
$$

can be written explicitly as follows. 
(i) $E^{2}>1$.

In this case it is convenient to use the notation $E=\cosh \alpha$. We have then

$$
\begin{aligned}
& t(\tau)=\frac{1}{H}\left[\operatorname{arctanh}\left(\frac{\tanh \left(\frac{1}{2} H \tau\right)+\sinh \alpha}{\cosh \alpha}\right)+\operatorname{arctanh}\left(\frac{\tanh \left(\frac{1}{2} H \tau\right)-\sinh \alpha}{\cosh \alpha}\right)\right] \\
& r(\tau)=\frac{\sinh \alpha}{H} \sinh (H \tau)
\end{aligned}
$$

(ii) $E^{2}=1$.

In this case the initial conditions (2.9) imply the trivial solution of a particle at rest at the origin

$$
r=0, \quad t=\tau .
$$

(iii) $E^{2}<1$.

In this case it is convenient to use the notation $E=\cos \alpha$. However $r(0)$ cannot be made to vanish but it should be always greater than a certain value $0<r_{*}=\sin \alpha / H<1 / H$. We have then

$$
\begin{aligned}
t(\tau) & =\frac{1}{H}\left\{\operatorname{arctanh}\left[\frac{1+\sin \alpha}{\cos \alpha} \tanh \left(\frac{1}{2} H \tau\right)\right]+\operatorname{arctanh}\left[\frac{1-\sin \alpha}{\cos \alpha} \tanh \left(\frac{1}{2} H \tau\right)\right]\right\} \\
r(\tau) & =\frac{\sin \alpha}{H} \cosh (H \tau) \equiv r_{*} \cosh (H \tau) .
\end{aligned}
$$

With a slight modification one can obtain the solution corresponding to more general initial data $x^{\alpha}(0)=x_{0}^{\alpha}$. For instance, for $r(0) \neq 0$ the solution (2.11) for $E^{2}=1$ becomes

$$
r=r_{0} e^{ \pm H \tau}, \quad t=\tau \mp \frac{1}{H} \ln \frac{N}{N_{0}} .
$$

For a later use we also introduce the Newman-Penrose [13 frame

$$
l=\frac{1}{N^{2}} \partial_{t}+\partial_{r}, \quad n=\frac{N^{2}}{2}\left(\frac{1}{N^{2}} \partial_{t}-\partial_{r}\right), \quad m=\frac{1}{\sqrt{2} r}\left(\partial_{\theta}+\frac{\mathrm{i}}{\sin \theta} \partial_{\phi}\right) .
$$

The non-vanishing spin coefficients are

$$
\rho=-\frac{1}{r}, \quad \mu=-\frac{N^{2}}{2 r}, \quad \alpha=-\beta=-\frac{\sqrt{2}}{4 r} \cot \theta, \quad \gamma=-\frac{H^{2} r}{2} .
$$

The Weyl scalars are all identically zero.

\section{Metric perturbations}

Let us consider the perturbations

$$
\tilde{g}_{\mu \nu}=g_{\mu \nu}+h_{\mu \nu}
$$

of de Sitter spacetime. Since the background metric (2.1) is static and spherically symmetric, we decompose the metric perturbation $h_{\mu \nu}$ in tensor harmonics and Fourier transform it with respect to time, as customary. We will use the Regge-Wheeler [9] gauge to simplify the form of the perturbation. 


\subsection{Electric multipoles}

The metric perturbation $h_{\mu \nu}$, for the electric multipoles, is given by

$$
\left\|h_{\mu \nu}\right\|=\left[\begin{array}{cccc}
N^{2} H_{0} & H_{1} & 0 & 0 \\
\operatorname{sym} & \frac{1}{N^{2}} H_{2} & 0 & 0 \\
\text { sym } & \text { sym } & r^{2} K & 0 \\
\text { sym } & \text { sym } & \text { sym } & r^{2} \sin ^{2} \theta K
\end{array}\right] \mathrm{e}^{-\mathrm{i} \omega t} Y_{l 0},
$$

where the symbol "sym" inherited from [9] indicates that the missing components of $h_{\mu \nu}$ should be found from the symmetry $h_{\mu \nu}=h_{\nu \mu}$ and the functions $Y_{l 0}$ are normalized spherical harmonics (see e.g. [14]) with azimuthal index $m=0$, defined by

$$
Y_{l 0}=\frac{1}{2} \sqrt{\frac{2 l+1}{\pi}} P_{l}(\cos \theta) .
$$

For $l \geq 2$, the system of radial equations we have to solve is the following (here $L \equiv l(l+1))$ :

$$
\begin{aligned}
& 0=H_{1}{ }^{\prime}+\frac{\mathrm{i} \omega}{N^{2}}(W+K)-\frac{2 r H^{2}}{N^{2}} H_{1}, \\
& 0=K^{\prime}-\frac{W}{r}-\frac{\mathrm{i} L}{2 \omega r^{2}} H_{1}+\frac{K}{r N^{2}}
\end{aligned}
$$

since

$$
H_{0}=H_{2} \equiv W
$$

together with the algebraic relation

$$
0=\frac{(L-2)}{r} W-\frac{\mathrm{i}}{\omega}\left(H^{2} L+2 \omega^{2}\right) H_{1}+\frac{2}{r N^{2}}\left(1+r^{2} \omega^{2}-N^{2} \frac{L}{2}\right) K .
$$

Let us introduce the dimensionless variables $x \equiv H r, \Omega \equiv \omega / H$, and set $H_{1} \equiv \mathrm{i} \tilde{H}_{1}$. Solving for $W$ in the constraint equation (3.6) and substituting into the system (3.4) we get (from now on a prime denotes differentiation with respect to $x$ )

$$
\begin{aligned}
\tilde{H}_{1}{ }^{\prime} & =\frac{\left(2 \Omega^{2}+3 L-4\right) x}{\left(1-x^{2}\right)(L-2)} \tilde{H}_{1}+\frac{2 \Omega\left[\left(\Omega^{2}+L-1\right) x^{2}+2-L\right]}{\left(1-x^{2}\right)^{2}(L-2)} K, \\
K^{\prime} & =-\frac{\left[2 x^{2}\left(L+2 \Omega^{2}\right)+L(L-2)\right]}{2(L-2) \Omega x^{2}} \tilde{H}_{1}-\frac{x\left(L+2 \Omega^{2}\right)}{\left(1-x^{2}\right)(L-2)} K,
\end{aligned}
$$

which can be solved in terms of Heun's functions (see Appendix C).

Figure 1 shows the behaviour of the metric perturbation functions $\tilde{H}_{1}, K$ and $W$ in terms of the rescaled radial variable $x$ for $l=2$ and different values of $\Omega$. The perturbative regime is generally lost very quickly. High frequency modes instead oscillate with small amplitudes over a wide range of the radial coordinate. The oscillations become very high when approaching the de Sitter horizon in the case of $\tilde{H}_{1}$ and $W$. The amplitude remains instead practically constant in the case of $K$. In fact, in the high frequency limit $\Omega \gg 1$ one finds

$$
K^{\prime \prime}+\frac{2 x}{1-x^{2}} K^{\prime}+\frac{\Omega^{2}}{\left(1-x^{2}\right)^{2}} K=0,
$$




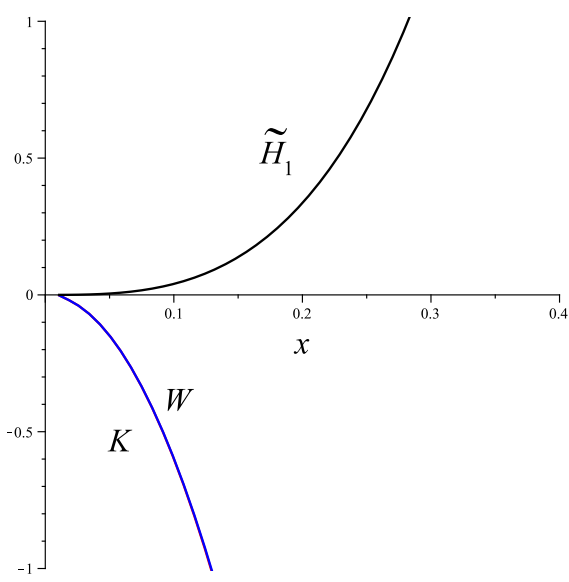

(a)

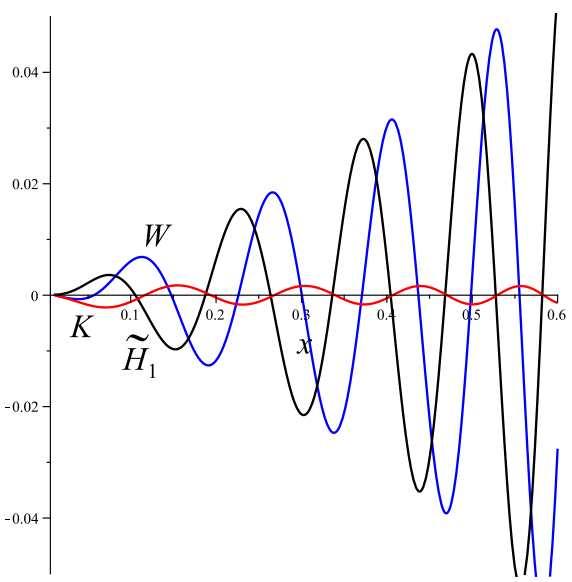

(b)

Figure 1. The behaviour of the metric perturbation functions $\tilde{H}_{1}, K$ and $W$ in the case of electric multipoles is shown in terms of the rescaled radial variable $x$ for $l=2$ and different values of $\Omega$. Fig. (a) corresponds to the choice $\Omega=1$ and the initial conditions are $\tilde{H}_{1}\left(x_{0}\right)=10^{-4}=K\left(x_{0}\right)$, with $x_{0}=10^{-2}$, so that $W\left(x_{0}\right) \approx 10^{-4}$. Fig. (b) corresponds to $\Omega=40$ and initial conditions $\tilde{H}_{1}\left(x_{0}\right)=10^{-4}=K\left(x_{0}\right)$, with $x_{0}=10^{-2}$, so that $W\left(x_{0}\right) \approx 7 \times 10^{-5}$. Larger values of $l$ correspond to a further growth of the perturbation functions.

or equivalently

$$
\frac{d^{2} K}{d z^{2}}+\Omega^{2} K=0
$$

having defined $z=\operatorname{arctanh} x$. The solution is then

$$
K=c_{1} \sin (\Omega \operatorname{arctanh} x)+c_{2} \cos (\Omega \operatorname{arctanh} x),
$$

where $c_{1}$ and $c_{2}$ are arbitrary constants.

A real solution for the metric can be obtained by considering $W$ and $K$ and $\tilde{H}_{1}$ as real. In this case the non-vanishing metric components are

$$
\begin{aligned}
g_{t t} & =N^{2}\left(1+W Y_{l 0} \cos \omega t\right), \\
g_{t r} & =\tilde{H}_{1} Y_{l 0} \sin \omega t, \\
g_{r r} & =-N^{-2}\left(1-W Y_{l 0} \cos \omega t\right), \\
g_{\theta \theta} & =-r^{2}\left(1-K Y_{l 0} \cos \omega t\right), \\
g_{\phi \phi} & =-r^{2} \sin ^{2} \theta\left(1-K Y_{l 0} \cos \omega t\right),
\end{aligned}
$$

so that

$$
\begin{aligned}
d s^{2}= & N^{2}\left(1+W Y_{l 0} \cos \omega t\right)\left(d t-\tilde{H}_{1} N^{-2} Y_{l 0} \sin \omega t d r\right)^{2} \\
& -N^{-2}\left(1-W Y_{l 0} \cos \omega t\right) d r^{2} \\
& -r^{2}\left(1-K Y_{l 0} \cos \omega t\right)\left(d \theta^{2}+\sin ^{2} \theta d \phi^{2}\right)
\end{aligned}
$$


to first order in the perturbation quantities. A natural orthonormal frame associated with this form of the metric is then

$$
\begin{aligned}
\Omega^{\hat{0}} & =N\left(1+\frac{1}{2} W Y_{l 0} \cos \omega t\right)\left(d t+\tilde{H}_{1} N^{-2} Y_{l 0} \sin \omega t d r\right) \\
& =N\left(1+\frac{1}{2} W Y_{l 0} \cos \omega t\right) d t+\tilde{H}_{1} N^{-1} Y_{l 0} \sin \omega t d r \\
\Omega^{\hat{r}} & =-N^{-1}\left(1-\frac{1}{2} W Y_{l 0} \cos \omega t\right) d r \\
\Omega^{\hat{\theta}} & =-r\left(1-\frac{1}{2} K Y_{l 0} \cos \omega t\right) d \theta \\
\Omega^{\hat{\phi}} & =-r \sin \theta\left(1-\frac{1}{2} K Y_{l 0} \cos \omega t\right) d \phi .
\end{aligned}
$$

One can then introduce a Newman-Penrose [13] frame in a standard way [15]

$l=\left(\Omega^{\hat{0}}+\Omega^{\hat{r}}\right) / \sqrt{2}, \quad n=\left(\Omega^{\hat{0}}-\Omega^{\hat{r}}\right) / \sqrt{2}, \quad m=\left(\Omega^{\hat{\theta}}+\mathrm{i} \Omega^{\hat{\phi}}\right) / \sqrt{2}$,

and conveniently rotate it according to $l \rightarrow l \sqrt{2} / N, n \rightarrow n N / \sqrt{2}$. We list here the non-vanishing first-order spin coefficients and Weyl scalars (hereafter $C \equiv \cos \omega t$ and $S \equiv \sin \omega t)$

$$
\begin{aligned}
\rho & =-\frac{1}{r}+\frac{1}{2 r}\left(r K^{\prime}-W\right) Y_{l 0} C-\frac{\omega}{2 N^{2}} K Y_{l 0} S \\
\mu & =-\frac{N^{2}}{2 r}+\frac{N^{2}}{4 r}\left(r K^{\prime}-W\right) Y_{l 0} C+\frac{\omega}{4} K Y_{l 0} S \\
\alpha & =-\frac{\sqrt{2}}{4 r} \cot \theta+\frac{\sqrt{2}}{8 r} K\left(\frac{d Y_{l 0}}{d \theta}-\cot \theta Y_{l 0}\right) C-\frac{\sqrt{2}}{8 r} \tilde{H}_{1} Y_{l 0} S \\
\beta & =-\alpha-\frac{\sqrt{2}}{4 r} \tilde{H}_{1} \frac{d Y_{l 0}}{d \theta} S \\
\gamma & =-\frac{H^{2} r}{2}-\frac{1}{8}\left(-N^{2} W^{\prime}+2 \omega \tilde{H}_{1}+2 r H^{2} W\right) Y_{l 0} C-\frac{\omega}{8} W Y_{l 0} S \\
\nu & =\frac{\sqrt{2} N^{2}}{8 r}\left(W C-\tilde{H}_{1} S\right) \frac{d Y_{l 0}}{d \theta} \\
\epsilon & =\frac{Y_{l 0}}{4 N^{2}}\left[\left(N^{2} W^{\prime}-2 \omega \tilde{H}_{1}\right) C+\omega W S\right] \\
\kappa & =-\frac{\sqrt{2}}{2 N^{2} r}\left(W C+\tilde{H}_{1} S\right) \frac{d Y_{l 0}}{d \theta}
\end{aligned}
$$

and

$$
\begin{aligned}
& \psi_{0}=-\frac{1}{2 N^{2} r^{2}}\left(W C+\tilde{H}_{1} S\right)_{2} Y_{l}^{0}{ }_{l} \\
& \psi_{1}=\frac{\sqrt{2}}{4 N^{2} r^{2}}\left[\frac{\left(\tilde{H}_{1} L N^{2}+2 r \omega K\right)}{2 r \omega} C+\left(\tilde{H}_{1} N^{2}+r \omega K\right) S\right] \frac{d Y_{l 0}}{d \theta}, \\
& \psi_{2}=\frac{L}{4 \omega r^{3}}\left(\tilde{H}_{1} N^{2}+r \omega K\right) Y_{l 0} C \\
& \psi_{3}=-\frac{\sqrt{2}}{8 r^{2}}\left[\frac{\left(\tilde{H}_{1} L N^{2}+2 r \omega K\right)}{2 r \omega} C-\left(\tilde{H}_{1} N^{2}+r \omega K\right) S\right] \frac{d Y_{l 0}}{d \theta} \\
& \psi_{4}=-\frac{N^{2}}{8 r^{2}}\left(W C-\tilde{H}_{1} S\right)_{2} Y_{l}^{0},
\end{aligned}
$$




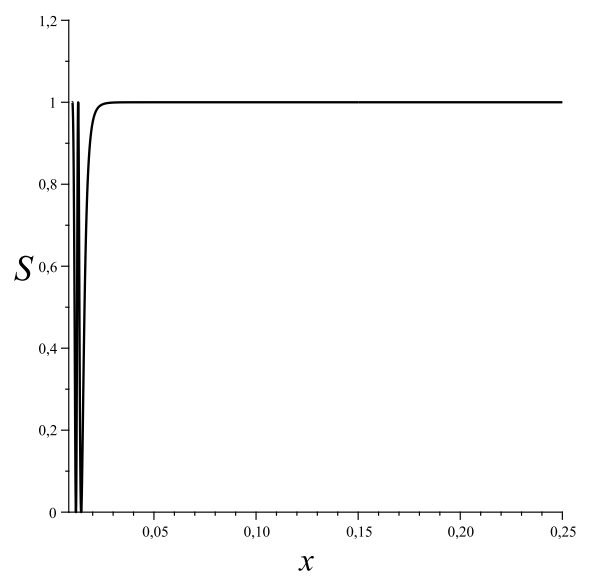

Figure 2. The behaviour of the spectral index $\mathcal{S}$ in the case of electric multipoles is shown in terms of the rescaled radial variable $x$ for fixed values of $t=\pi / 4$, $\theta=\pi / 2, l=2$ and $\Omega=1$. The choice of initial conditions is the same as in Fig. 1 (a). The variation of radial coordinate is limited to the range where the perturbative regime holds.

where

$$
{ }_{2} Y^{0}{ }_{l}=2 \frac{d Y_{l 0}}{d \theta} \cot \theta+L Y_{l 0} .
$$

Note that the first-order Ricci scalar coincides with the zeroth-order one.

It is useful to introduce the speciality index $\mathcal{S}$, which is a suitable combination of Weyl scalars defined by

$$
\mathcal{S}=\frac{27 J^{2}}{I^{3}}=27 \frac{\left(\psi_{0} \psi_{2} \psi_{4}-\psi_{2}^{3}\right)^{2}}{\left(\psi_{0} \psi_{4}+3 \psi_{2}^{2}\right)^{3}}
$$

and has the value 1 for algebraically special spacetimes. Note that the imaginary part of $\mathcal{S}$ turns out to be identically vanishing, so that it is a purely real quantity. Its behaviour as a function of the rescaled radial coordinate $x$ for fixed values of $t$ and $\theta$ is shown in Fig. 2 .

\subsection{Magnetic multipoles}

The metric perturbation $h_{\mu \nu}$, for magnetic multipoles, is given by

$$
\left\|h_{\mu \nu}\right\|=\left[\begin{array}{cccc}
0 & 0 & 0 & h_{0} \\
\text { sym } & 0 & 0 & h_{1} \\
\text { sym } & \text { sym } & 0 & 0 \\
\text { sym } & \text { sym } & \text { sym } & 0
\end{array}\right] \mathrm{e}^{-\mathrm{i} \omega t} \sin \theta \frac{d Y_{l 0}}{d \theta} .
$$

For $l \geq 2$, the system of radial equations we have to solve is

$$
\begin{aligned}
& 0=h_{0}{ }^{\prime}-\frac{2 h_{0}}{r}+\mathrm{i} h_{1}\left[\omega-\frac{N^{2}}{r^{2}} \frac{(L-2)}{\omega}\right], \\
& 0=h_{1}{ }^{\prime}-\frac{2 r H^{2}}{N^{2}} h_{1}-\frac{\mathrm{i} \omega}{N^{4}} h_{0},
\end{aligned}
$$




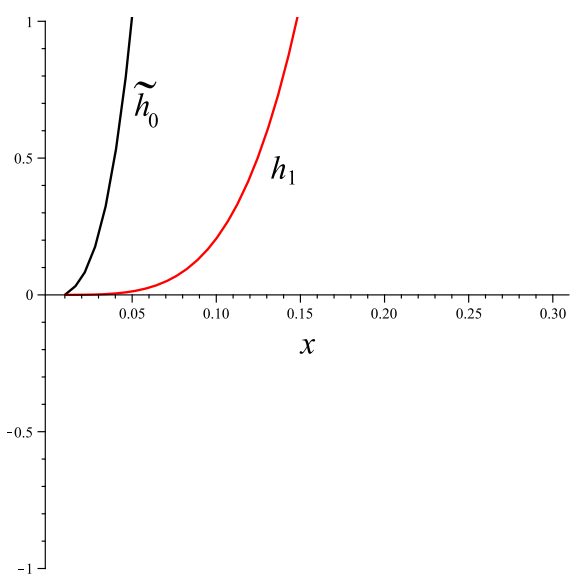

(a)

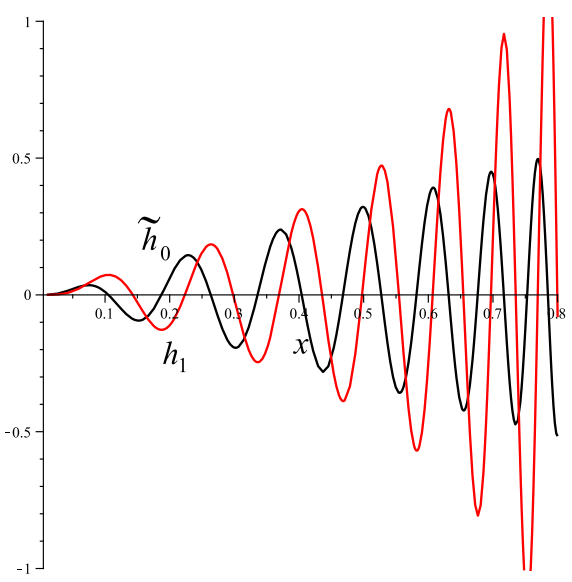

(b)

Figure 3. The behaviour of the metric perturbation functions $\tilde{h}_{0}$ and $h_{1}$ in the case of magnetic multipoles is shown in terms of the rescaled radial variable $\underset{\sim}{x}$ for $l=2$ and different values of $\Omega$. The initial conditions are chosen so that $\tilde{h}_{0}\left(x_{0}\right)=10^{-4}=h_{1}\left(x_{0}\right)$, with $x_{0}=10^{-2}$. Figures (a) and (b) correspond to $\Omega=1$ and $\Omega=40$, respectively.

where again $L \equiv l(l+1)$.

By introducing the dimensionless variables $x \equiv H r, \Omega \equiv \omega / H$ as above and setting $h_{0} \equiv i \tilde{h}_{0}$, the system (3.21) becomes

$$
\begin{aligned}
\tilde{h}_{0}{ }^{\prime} & =\frac{2}{x} \tilde{h}_{0}-\frac{\left[\left(\Omega^{2}+L-2\right) x^{2}+2-L\right]}{\Omega x^{2}} h_{1}, \\
h_{1}{ }^{\prime} & =\frac{\Omega}{\left(1-x^{2}\right)^{2}} \tilde{h}_{0}+\frac{2 x}{1-x^{2}} h_{1},
\end{aligned}
$$

which can be solved in terms of Heun's functions (see appendix C).

Figure 3 shows the behaviour of the metric perturbation functions $\tilde{h}_{0}$ and $h_{1}$ in terms of the rescaled radial variable $x$ for $l=2$ and different values of $\Omega$. The same considerations holding for electric multipoles apply also in this case.

A real solution for the metric can be obtained by considering $\tilde{h}_{0}$ and $h_{1}$ as real. In this case the non-vanishing metric components are

$$
\begin{aligned}
& g_{t t}=N^{2}, \quad g_{t \phi}=\tilde{h}_{0} \sin \omega t \sin \theta \frac{d Y_{l 0}}{d \theta}, \quad g_{r \phi}=h_{1} \cos \omega t \sin \theta \frac{d Y_{l 0}}{d \theta}, \\
& g_{r r}=-N^{-2}, \quad g_{\theta \theta}=-r^{2}, \quad g_{\phi \phi}=-r^{2} \sin ^{2} \theta,
\end{aligned}
$$

so that

$$
d s^{2}=d s_{(\mathrm{dS})}^{2}+2 \sin \theta \frac{d Y_{l 0}}{d \theta}\left(\tilde{h}_{0} \sin \omega t d \phi+2 h_{1} \cos \omega t d r\right) d t,
$$

to first order in the perturbation quantities. A natural orthonormal frame associated with this form of the metric is then

$$
\Omega^{\hat{0}}=N d t+N^{-1} \sin \theta \frac{d Y_{l 0}}{d \theta} \tilde{h}_{0} \sin \omega t d \phi
$$


de Sitter spacetime: effects of metric perturbations on geodesics

$$
\begin{aligned}
& \Omega^{\hat{r}}=-N^{-1} d r+N \sin \theta \frac{d Y_{l 0}}{d \theta} h_{1} \cos \omega t d \phi, \\
& \Omega^{\hat{\theta}}=-r d \theta, \quad \Omega^{\hat{\phi}}=-r \sin \theta d \phi .
\end{aligned}
$$

One can then introduce a Newman-Penrose frame in a standard way, as in Eq. (3.15). We find that the non-vanishing first-order spin coefficients, Weyl scalars and Ricci terms are as follows:

$$
\begin{aligned}
\rho= & -\frac{1}{r}-\mathrm{i} \frac{L}{2 N^{2} r^{2}}\left[N^{2} h_{1} C+\tilde{h}_{0} S\right] Y_{l 0}, \\
\mu= & -\frac{N^{2}}{2 r}+\mathrm{i} \frac{L}{4 r^{2}}\left[N^{2} h_{1} C-\tilde{h}_{0} S\right] Y_{l 0}, \\
\alpha= & -\frac{\sqrt{2}}{4 r} \cot \theta-\mathrm{i} \frac{\sqrt{2}}{4 r^{2}}\left[\left(1-2 N^{2}\right) h_{1} C+\frac{2 \omega r \tilde{h}_{0}+N^{4}(L-2) h_{1}}{2 \omega r N^{2}} S\right] \frac{d Y_{l 0}}{d \theta}, \\
\beta= & -\alpha+\mathrm{i} \frac{\sqrt{2} N^{2}}{2 r^{2}} h_{1} \frac{d Y_{l 0}}{d \theta} C, \\
\gamma= & -\frac{H^{2} r}{2}+\mathrm{i} \frac{L}{8 r^{2}}\left[N^{2} h_{1} C-\tilde{h}_{0} S\right] Y_{l 0}, \\
\nu= & \mathrm{i} \frac{\sqrt{2}}{4 r}\left\{\left[N^{2} H^{2} r h_{1}+\omega \tilde{h}_{0}\right] C\right. \\
& \left.-\frac{1}{2 \omega r^{2}}\left[2 \omega r \tilde{h}_{0}+N^{2}\left(N^{2}(L-2)-2 \omega^{2} r^{2}\right) h_{1}\right] S\right\} \frac{d Y_{l 0}}{d \theta}, \\
\epsilon= & -\mathrm{i} \frac{L}{4 N^{2} r^{2}}\left[N^{2} h_{1} C+\tilde{h}_{0} S\right] Y_{l 0}, \\
\kappa= & \frac{\sqrt{2}}{N^{4} r}\left\{\left[N^{2} H^{2} r h_{1}+\omega \tilde{h}_{0}\right] C\right. \\
& \left.+\frac{1}{2 \omega r^{2}}\left[2 \omega r \tilde{h}_{0}+N^{2}\left(N^{2}(L-2)-2 \omega^{2} r^{2}\right) h_{1}\right] S\right\} \frac{d Y_{l 0}}{d \theta},
\end{aligned}
$$

and

$$
\begin{aligned}
\psi_{0}= & \mathrm{i} \frac{1}{r^{2} N^{4}}\left\{\left[N^{2} H^{2} r h_{1}+\omega \tilde{h}_{0}\right] C\right. \\
& \left.+\frac{1}{2 \omega r^{2}}\left[2 \omega r \tilde{h}_{0}+N^{2}\left(N^{2}(L-2)-2 \omega^{2} r^{2}\right) h_{1}\right] S\right\}{ }_{2} Y_{l}^{0}, \\
\psi_{1}= & \mathrm{i} \frac{\sqrt{2}(L-2)}{4 r^{3}}\left[h_{1} C-\frac{1}{\omega r N^{2}}\left[N^{4} h_{1}-\omega r \tilde{h}_{0}\right] S\right] \frac{d Y_{l 0}}{d \theta}, \\
\psi_{2}= & -\mathrm{i} \frac{N^{2}}{4 \omega r^{4}} L(L-2) h_{1} Y_{l 0} S, \\
\psi_{3}= & \mathrm{i} \frac{\sqrt{2} N^{2}(L-2)}{8 r^{3}}\left[h_{1} C+\frac{1}{\omega r N^{2}}\left[N^{4} h_{1}-\omega r \tilde{h}_{0}\right] S\right] \frac{d Y_{l 0}}{d \theta}, \\
\psi_{4}= & -\mathrm{i} \frac{1}{4 r^{2}}\left\{\left[N^{2} H^{2} r h_{1}+\omega \tilde{h}_{0}\right] C\right. \\
& \left.-\frac{1}{2 \omega r^{2}}\left[2 \omega r \tilde{h}_{0}+N^{2}\left(N^{2}(L-2)-2 \omega^{2} r^{2}\right) h_{1}\right] S\right\}{ }_{2} Y_{l}^{0} .
\end{aligned}
$$

The behaviour of the speciality index (3.19) as a function of the rescaled radial coordinate $x$ for fixed values of $t$ and $\theta$ is shown in Fig. 4. Note that $\mathcal{S}$ is purely real also in this case. 


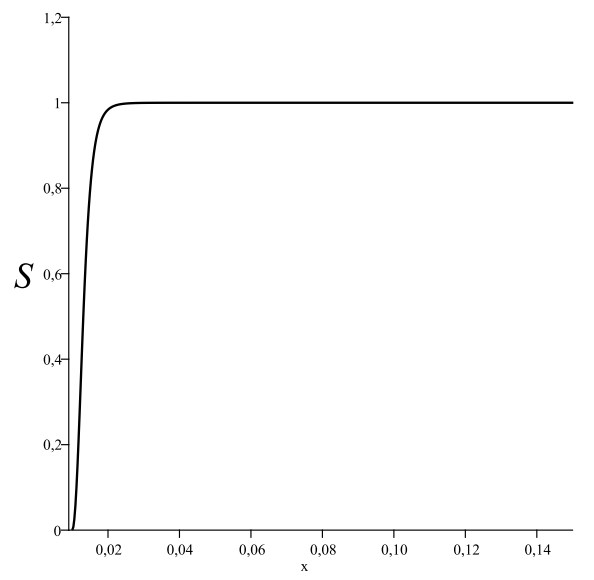

Figure 4. The behaviour of the spectral index $\mathcal{S}$ in the case of magnetic multipoles is shown in terms of the rescaled radial variable $x$ for fixed values of $t=\pi / 4, \theta=\pi / 2, l=2$ and $\Omega=1$. The choice of initial conditions is the same as in Fig. 3 (a). The variation of radial coordinate is limited to the range where the perturbative regime holds.

\section{Perturbations of radial geodesics}

We are now in a position to consider the perturbations

$$
\tilde{U}=U_{\text {geo }}+u
$$

to the radial geodesic (2.2). We are interested in finding the solution for $u$ such that the path is geodesic in the perturbed gravitational field, i.e. $\nabla_{\tilde{U}} \tilde{U}=0$, to first order in the perturbation.

\subsection{Electric multipoles}

The perturbed 4-velocity is given by

$$
u=\mathrm{e}^{-\mathrm{i} \omega t}\left[-\frac{\Gamma_{1}}{N^{2}} Y_{l 0} \partial_{t}+\zeta_{1} N^{2} Y_{l 0} \partial_{r}+\frac{\xi_{1}}{r^{2}} \frac{d Y_{l 0}}{d \theta} \partial_{\theta}\right],
$$

where $\Gamma_{1}, \zeta_{1}$ and $\xi_{1}$ are functions of $r$. Real solutions for $u$ require

$$
\begin{aligned}
& \mathrm{e}^{-\mathrm{i} \omega t} \xi_{1}=A_{1} \cos \omega t+A_{2} \sin \omega t \\
& \mathrm{e}^{-\mathrm{i} \omega t} \zeta_{1}=\frac{1}{E \zeta_{0}}\left[\left(B_{1}+\frac{N^{2}}{2} W\right) \cos \omega t+B_{2} \sin \omega t\right] \\
& \mathrm{e}^{-\mathrm{i} \omega t} \Gamma_{1}=-\frac{1}{E}\left[\left(B_{1}+E^{2} W\right) \cos \omega t+\left(B_{2}+E \Gamma_{0} \zeta_{0} \tilde{H}_{1}\right) \sin \omega t\right]
\end{aligned}
$$

where the functions $A_{1}, A_{2}$ and $B_{1}, B_{2}$ satisfy the systems of equations

$$
\begin{aligned}
\frac{d A_{1}}{d r} & =-\frac{\omega}{\zeta_{0}}\left(A_{2}-\frac{2 E^{2}-N^{2}}{2 E \omega} W\right), \\
\frac{d A_{2}}{d r} & =\frac{\omega}{\zeta_{0}}\left(A_{1}+\frac{\Gamma_{0} \zeta_{0}}{\omega} \tilde{H}_{1}\right),
\end{aligned}
$$


and

$$
\begin{aligned}
& \frac{d B_{1}}{d r}=-\frac{\omega}{\zeta_{0}}\left(B_{2}+E \Gamma_{0} \zeta_{0} \tilde{H}_{1}\right), \\
& \frac{d B_{2}}{d r}=\frac{\omega}{\zeta_{0}}\left(B_{1}+\frac{2 E^{2}-N^{2}}{2} W\right),
\end{aligned}
$$

respectively, which can be integrated numerically.

The behavior of the perturbed velocity functions (4.3) is shown in Fig. 5 in terms of the rescaled radial variable $x$ for different values of $t$ and fixed values of $l, \Omega$ and E.

\subsection{Magnetic multipoles}

The perturbed 4-velocity is given by

$$
u=\mathrm{e}^{-\mathrm{i} \omega t} \frac{\xi_{1}}{r^{2} \sin \theta} \frac{d Y_{l 0}}{d \theta} \partial_{\phi},
$$

where $\xi_{1}$ is a function of $r$. Real solutions for $u$ require

$$
\mathrm{e}^{-\mathrm{i} \omega t} \xi_{1}=A_{1} \cos \omega t+A_{2} \sin \omega t
$$

where the functions $A_{1}, A_{2}$ satisfy the system of equations

$$
\begin{aligned}
& \frac{d A_{1}}{d r}=-\frac{\omega}{\zeta_{0}}\left[A_{2}+\frac{2 E^{2}-N^{2}}{E N^{2}}\left(\tilde{h}_{0}+\frac{r H^{2} N^{2}}{\omega} h_{1}\right)\right], \\
& \frac{d A_{2}}{d r}=\frac{\omega}{\zeta_{0}}\left\{A_{1}-\frac{2 \Gamma_{0} \zeta_{0}}{\omega r N^{2}}\left[\tilde{h}_{0}-\omega r N^{2}\left(1-\frac{N^{2}(L-2)}{2 \omega^{2} r^{2}}\right) h_{1}\right]\right\},
\end{aligned}
$$

which can be integrated numerically.

The behavior of the perturbed velocity function (4.7) is shown in Fig. 6 in terms of the rescaled radial variable $x$ for different values of $t$ and fixed values of $l, \Omega$ and $E$.

\section{Teukolsky equation}

In this section we study gauge- and tetrad-invariant first-order massless perturbations of any spin $(s= \pm 2$ for gravitational waves, $s= \pm 1$ for electromagnetic waves, and $s= \pm 1 / 2$ for neutrinos) in the de Sitter background following the approach of Teukolsky [10]. Although this problem have been already addressed in the literature (see, e.g., Ref. [16, where the perturbation equations for fields of different spin have been solved by using the technique of Debye potentials), we are are interested here in investigating the existence of closed-form, i.e. Liouvillian, solutions to the perturbation equations.

The Teukolsky master equation, in this case, reads as

$$
\begin{aligned}
0=\{ & \frac{1}{2 N^{2}} \partial_{t t}-\frac{N^{2}}{2} \partial_{r r}-\frac{1}{2 r^{2}} \partial_{\theta \theta}-\frac{1}{2 r^{2} \sin ^{2} \theta} \partial_{\phi \phi} \\
& +\frac{s}{r N^{2}} \partial_{t}+\frac{(s+1)\left(1-2 N^{2}\right)}{r} \partial_{r}-\frac{\cot \theta}{2 r^{2}} \partial_{\theta} \\
& \left.-\frac{i s \cos \theta}{r^{2} \sin ^{2} \theta} \partial_{\phi}+\frac{1}{2 r^{2}}\left[(1+s)\left[3 s+2-2 N^{2}(2 s+1)\right]+\frac{s^{2}}{\sin ^{2} \theta}\right]\right\} \psi
\end{aligned}
$$




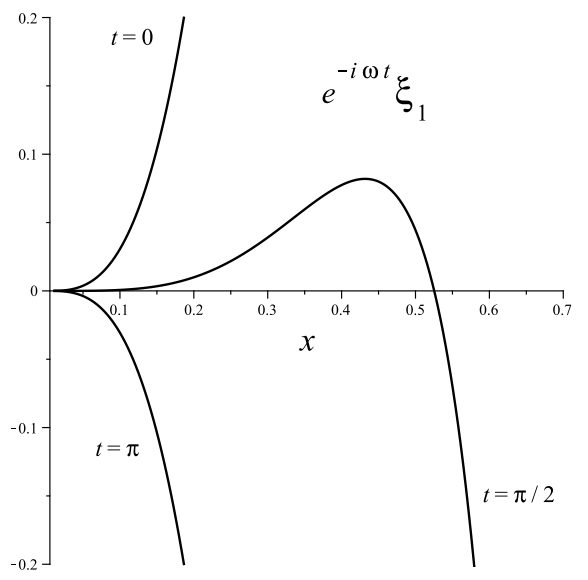

(a)

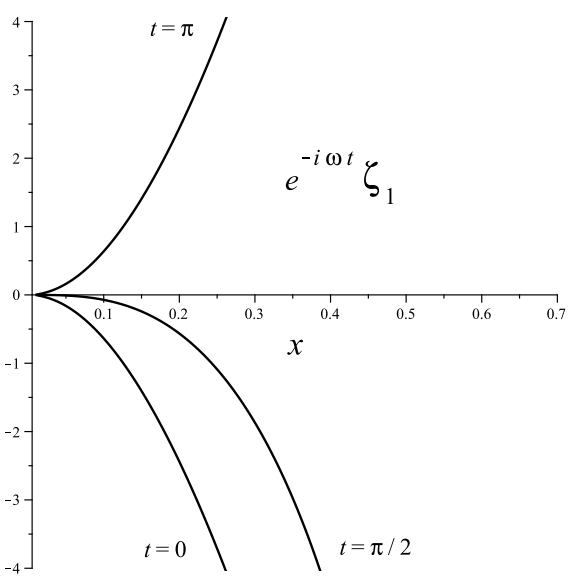

(b)

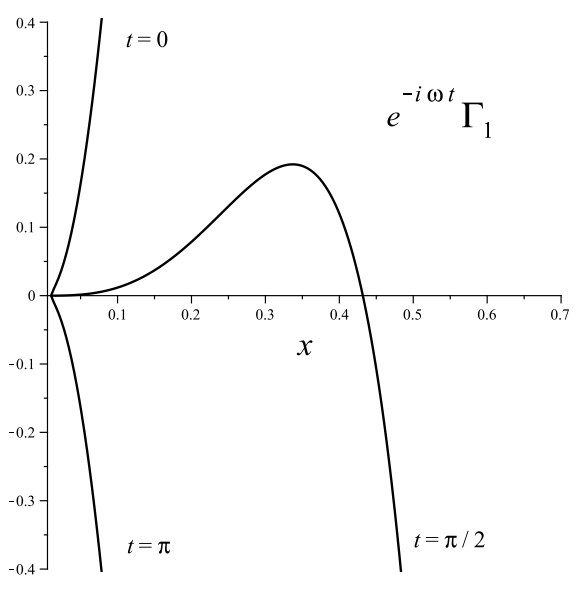

(c)

Figure 5. The behavior of the perturbed velocity functions $\mathrm{e}^{-\mathrm{i} \omega t} \xi_{1}, \mathrm{e}^{-\mathrm{i} \omega t} \zeta_{1}$ and $\mathrm{e}^{-\mathrm{i} \omega t} \Gamma_{1}$ in the case of electric multipoles is shown in terms of the rescaled radial variable $x$ for different values of $t$ and the same choice of parameters as well as initial conditions for the perturbed metric functions as in Fig. 1)(a). The functions $A_{1}, A_{2}$ and $B_{1}, B_{2}$ are taken instead identically vanishing at the initial point. The unperturbed geodesic radial motion is directed outwards with energy parameter $E=1.1$. Note that in the high frequency limit the perturbed velocity functions exhibit instead an oscillating behavior similar to that of the perturbed metric functions, the amplitude of $\mathrm{e}^{-\mathrm{i} \omega t} \xi_{1}$ at fixed distance being about $10^{2}$ smaller than that associated with $\zeta_{1}$ and $\Gamma_{1}$. 


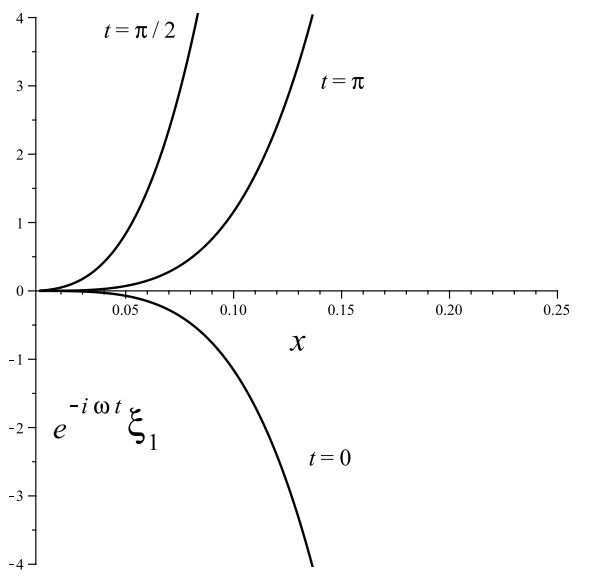

Figure 6. The behavior of the perturbed velocity function $e^{-i \omega t} \xi_{1}$ in the case of magnetic multipoles is shown in terms of the rescaled radial variable $x$ for different values of $t$ and the same choice of parameters as well as initial conditions for the perturbed metric functions as in Fig. 3 (a). The functions $A_{1}, A_{2}$ are taken instead identically vanishing at the initial point. The unperturbed geodesic radial motion is directed outwards with energy parameter $E=1.1$. Note that in the high frequency limit the perturbed velocity function exhibits instead an oscillating behavior similar to that of the perturbed metric functions.

Note that this equation is solved by $\psi=\psi_{0}$ with $s=2$ and by $\psi=\psi_{4} / \rho^{4}$ with $s=-2$, where $\psi_{0}$ and $\psi_{4}$ are the perturbed Weyl scalars given by Eqs. (3.17) and (3.28) for electric and magnetic multipoles, respectively. Therefore, the latter equations provide a link between the perturbed metric functions and the solutions to the Teukolsky equation.

Let us now study the Teukolsky equation in completely general form, relaxing the assumption of azimuthal symmetry. The master equation (5.1) admits separable solutions of the form

$$
\psi(t, r, \theta, \phi)=\mathrm{e}^{-\mathrm{i} \omega t} \mathrm{e}^{\mathrm{i} m \phi} R(r) S(\theta),
$$

where $\omega>0$ is the wave frequency and $m$ is the azimuthal separation constant.

The angular function satisfies the equation of the spin-weighted spherical harmonics, i.e.

where

$$
\frac{1}{\sin \theta} \frac{\mathrm{d}}{\mathrm{d} \theta}\left(\sin \theta \frac{\mathrm{d} S(\theta)}{\mathrm{d} \theta}\right)+V_{(\text {ang })}(\theta) S(\theta)=0,
$$

$$
V_{\text {(ang) }}(\theta)=L-s^{2}-\frac{(s \cos \theta+m)^{2}}{\sin ^{2} \theta},
$$

and the separation constant has been chosen according to Teukolsky.

The radial equation can be cast in the form

$$
Q^{-s} \frac{\mathrm{d}}{\mathrm{d} r}\left(Q^{s+1} \frac{\mathrm{d} R(r)}{\mathrm{d} r}\right)+V_{(\mathrm{rad})}(r) R(r)=0,
$$

where $Q=r^{2} N^{2}$ and

$V_{(\mathrm{rad})}(r)=\frac{1}{N^{4}}\left\{\omega^{2}+\frac{2 \mathrm{i} \omega s}{r}-\frac{N^{2}}{r^{2}}[L+(1+s)(3 s+2)]+2 \frac{N^{4}}{r^{2}}(1+s)(2 s+1)\right\}$. 


\subsection{Liouvillian solutions to the radial equation}

The general solution to the radial part of the Teukolsky equation can be expressed in terms of Heun functions. We are interested here in investigating the existence of closed-form, i.e. Liouvillian, solutions to the radial equation (5.5) governing the perturbations. Roughly speaking, the set of Liouvillian functions includes the usual elementary functions such as exponential, trigonometric, logarithmic functions, etc., but not generic hypergeometric functions or other special functions. We apply the well-known Kovacic algorithm [17 outlined in appendix D.

The radial equation (5.5) can be converted in normal form, i.e.

$$
\frac{\mathrm{d}^{2} y(r)}{\mathrm{d} r^{2}}=W_{(\mathrm{rad})}(r) y(r)
$$

by the scaling

$$
R=y Q^{-(1+s) / 2},
$$

where the function $W_{(\mathrm{rad})}$ is given by

$$
W_{(\mathrm{rad})}(r)=\frac{c_{0}}{r^{2}}+\frac{c_{H}^{-}}{\left(r-r_{H}\right)^{2}}+\frac{c_{H}^{+}}{\left(r+r_{H}\right)^{2}}+\frac{d_{0}}{r}+\frac{d_{H}^{-}}{\left(r-r_{H}\right)}+\frac{d_{H}^{+}}{\left(r+r_{H}\right)},
$$

with parameters

$$
\begin{aligned}
& c_{0}=L, \quad c_{H}^{ \pm}=\frac{1}{4}\left[\left(s \pm \mathrm{i} \omega r_{H}\right)^{2}-1\right], \\
& d_{0}=-2 \mathrm{i} \omega s, \quad d_{H}^{ \pm}=\mp \frac{1}{4 r_{H}}\left[\omega^{2} r_{H}^{2} \mp 4 \mathrm{i} \omega r_{H} s-s^{2}-2 L+1\right] .
\end{aligned}
$$

Let us discuss in detail the case of type 1 solutions (see again appendix D). For all possible pole structures of (5.9), all functions $\tilde{\Omega}$ generated by the Kovacic algorithm have the form

$$
\tilde{\Omega}=\frac{\alpha_{0}}{r}+\frac{\alpha_{H}^{+}}{\left(r+r_{H}\right)}+\frac{\alpha_{H}^{-}}{\left(r-r_{H}\right)},
$$

where

$$
\alpha_{0}=\frac{1}{2}\left[1+\delta_{0} \sqrt{1+4 c_{0}}\right], \quad \alpha_{H}^{ \pm}=\frac{1}{2}\left[1+\delta_{H}^{ \pm} \sqrt{1+4 c_{H}^{ \pm}}\right],
$$

the quantities $\delta_{0}, \delta_{H}^{+}$and $\delta_{H}^{-}$taking independently values \pm 1 .

The necessary conditions for type 1 solutions are fulfilled if the following constraint is imposed:

$$
d_{0}+d_{H}^{+}+d_{H}^{-}=0
$$

implying that the order of the pole at infinity is 2 .

The algorithmic condition $d=n$ is given by

$$
\alpha_{\infty}-\alpha_{0}-\alpha_{H}^{+}-\alpha_{H}^{-}=n,
$$

where

$$
\alpha_{\infty}=\frac{1}{2}\left[1+\delta_{\infty} \sqrt{1+4\left(c_{0}+c_{H}^{+}+c_{H}^{-}\right)}\right], \quad \delta_{\infty}= \pm 1 .
$$

For every pole structure, we construct the Liouvillian solutions to Eq. (5.7) by deriving a consistent recursion relation which generates the finite set of coefficients in the polynomial $\mathcal{P}$. To obtain a recursion relation with the fewest number of terms 
we take, without loss of generality, $\mathcal{P}$ to be expanded about a pole of non-vanishing order, say $r=0$, as follows:

$$
\mathcal{P}=\sum_{k=0}^{n} a_{k} r^{k}, \quad a_{n} \neq 0 .
$$

After substituting this expression for $\mathcal{P}$ into Eq. (D.2) we obtain in the general case, when all three poles are present, the recursion relation

$$
\begin{aligned}
0= & {\left[(k-1)\left(k-2-2 n+2 \alpha_{\infty}\right)-r_{H}\left(g_{H}^{+}-g_{H}^{-}\right)\right] a_{k-1} } \\
& -r_{H}\left[2 k\left(\alpha_{H}^{+}-\alpha_{H}^{-}\right)-r_{H}\left(g_{H}^{+}+g_{H}^{-}\right)\right] a_{k} \\
& -(k+1) r_{H}^{2}\left(k+2 \alpha_{0}\right) a_{k+1}, \quad k=0, \ldots, n+1,
\end{aligned}
$$

where

$$
\begin{aligned}
& g_{0}=-d_{0}+2 \frac{\alpha_{0}}{r_{H}}\left(\alpha_{H}^{+}-\alpha_{H}^{-}\right), \\
& g_{H}^{ \pm}=-d_{H}^{ \pm} \mp \frac{\alpha_{H}^{ \pm}}{r_{H}}\left(2 \alpha_{0}+\alpha_{H}^{\mp}\right),
\end{aligned}
$$

with the understanding that $a_{i}=0$ if $i \leq-1$ or $i \geq n+1$.

Liouvillian solutions (D.3) to Eq. (5.7) are thus given by

$$
y=\mathcal{P} r^{\alpha_{0}}\left(r+r_{H}\right)^{\alpha_{H}^{+}}\left(r_{H}-r\right)^{\alpha_{H}^{-}}
$$

implying that

$$
R=\mathcal{P} r_{H}^{1+s} r^{\alpha_{0}-\frac{1+s}{2}}\left(r+r_{H}\right)^{\alpha_{H}^{+}-\frac{1+s}{2}}\left(r_{H}-r\right)^{\alpha_{H}^{-}-\frac{1+s}{2}} .
$$

\section{Concluding remarks}

The present work has been motivated by a previous study on gravitational waves about de Sitter backgrounds [18, which is relevant for the stochastic background emerging from inflation, and by the analysis in [19] of gravitational waves and their geodesics. We have solved the gravitational perturbation problem in the static region of the de Sitter spacetime between the origin and the cosmological horizon by using the Regge-Wheeler formalism. The set of perturbation equations has been reduced to a single second order differential equation of the Heun-type for both electric and magnetic multipoles. We have then investigated the geodesic motion in the perturbed gravitational field. In particular, an initially radial geodesic in the unperturbed spacetime cannot remain radial as a result of the perturbation. The deviation from radial motion has been shown to occur in both polar and azimuthal directions. Finally, we have investigated gauge- and tetrad-invariant first-order massless perturbations of any spin following the approach of Teukolsky. The general solution to the radial part of the Teukolsky equation can be expressed in terms of Heun functions. However, we have shown that there also exists in addition a class of closed-form, i.e. Liouvillian, solutions. 
de Sitter spacetime: effects of metric perturbations on geodesics

Appendix A. Electric multipoles: $l=0,1$

Appendix A.1. $l=0$

The system of perturbation equations in the case $l=0$ is given by

$$
\begin{aligned}
& 0=H_{0}{ }^{\prime}-\frac{2 \omega}{N^{2}} \tilde{H}_{1}-\frac{r H^{2}}{N^{2}} H_{2}-\frac{r}{N^{4}}\left(\omega^{2}+H^{2}\right) K, \\
& 0=K^{\prime}-\frac{H_{2}}{r}+\frac{K}{r N^{2}} .
\end{aligned}
$$

We can set

$$
H_{0}=H_{2} \equiv W, \quad \tilde{H}_{1}=0,
$$

without loss of generality, so that Eq. A.1 reduces to

$$
\begin{aligned}
& 0=W^{\prime}-\frac{r H^{2}}{N^{2}} W-\frac{r}{N^{4}}\left(\omega^{2}+H^{2}\right) K, \\
& 0=K^{\prime}-\frac{W}{r}+\frac{K}{r N^{2}} .
\end{aligned}
$$

In terms of the dimensionless variable $x \equiv H r$ the corresponding solution is

$$
\begin{aligned}
& K=\frac{\sqrt{1-x^{2}}}{x}\left[c_{1}\left(\frac{1-x}{1+x}\right)^{\sqrt{1+\Omega^{2}} / 2}+c_{2}\left(\frac{1-x}{1+x}\right)^{-\sqrt{1+\Omega^{2}} / 2}\right], \\
& W=\frac{\sqrt{1+\Omega^{2}}}{\sqrt{1-x^{2}}}\left[-c_{1}\left(\frac{1-x}{1+x}\right)^{\sqrt{1+\Omega^{2}} / 2}+c_{2}\left(\frac{1-x}{1+x}\right)^{-\sqrt{1+\Omega^{2}} / 2}\right]
\end{aligned}
$$

where $\Omega \equiv \omega / H$.

\section{Appendix A.2. $l=1$}

The system of perturbation equations in the case $l=1$ is given by

$$
\begin{aligned}
& 0=H_{0}{ }^{\prime}-\frac{H_{0}}{r N^{2}}+\frac{\omega^{2} r}{N^{4}} K-\frac{r H^{2}}{N^{2}} H_{2}, \\
& 0=K^{\prime}-\frac{H_{2}}{r},
\end{aligned}
$$

plus the algebraic relation

$$
\tilde{H}_{1}=\frac{\omega r}{N^{2}} K
$$

We can set

$$
H_{0}=H_{2} \equiv W
$$

so that Eq. A.5 becomes

$$
\begin{aligned}
& 0=W^{\prime}+\frac{\omega^{2} r}{N^{4}} K-\frac{\left(1+H^{2} r^{2}\right)}{r N^{2}} W, \\
& 0=K^{\prime}-\frac{W}{r} .
\end{aligned}
$$

In terms of the dimensionless variable $x \equiv H r$ the corresponding solution is

$$
\begin{aligned}
& K=c_{1} \sin (\Omega \operatorname{arctanh} x)+c_{2} \cos (\Omega \operatorname{arctanh} x), \\
& W=\frac{\Omega x}{\left(1-x^{2}\right)}\left[c_{1} \cos (\Omega \operatorname{arctanh} x)-c_{2} \sin (\Omega \operatorname{arctanh} x)\right] .
\end{aligned}
$$




\section{Appendix B. Magnetic multipoles: $l=1$}

The system of perturbation equations in the case $l=1$ reduces to

$$
0=\tilde{h}_{0}{ }^{\prime}-\frac{2 \tilde{h}_{0}}{r}+\omega h_{1}
$$

Without loss of generality we can set $h_{1}=0$, so that the previous equation becomes

$$
0=\tilde{h}_{0}{ }^{\prime}-\frac{2 \tilde{h}_{0}}{r},
$$

whose solution is simply

$$
\tilde{h}_{0}=c_{1} r^{2} \text {. }
$$

\section{Appendix C. Decoupling the perturbation equations}

The system of perturbation equations of both parity can be reduced to a secondorder differential equation for a single perturbation function, the remaining ones being determined either algebraically or by differentiation. Such a master equation can be cast in general in the form of a Heun equation [20, 21.

$$
\frac{d^{2} y}{d z^{2}}+\left(\frac{\gamma}{z}+\frac{\delta}{z-1}+\frac{\epsilon}{z-a}\right) \frac{d y}{d z}+\frac{\alpha \beta-q}{z(z-1)(z-a)} y=0,
$$

where $\{\alpha, \beta, \gamma, \delta, \epsilon, a, q\}$ are complex arbitrary parameters such that $\gamma+\delta+\epsilon=\alpha+\beta+1$ and $a \neq 0,1$. This equation has four regular singular points, at $\{0,1, a, \infty\}$.

This is a generalization of the hypergeometric equation. In fact, in the special case where $\epsilon=0$ and $q=\alpha \beta a$, Eq. (C.1) becomes

$$
z(z-1) \frac{d^{2} y}{d z^{2}}+[(\alpha+\beta+1)-\gamma] \frac{d y}{d z}+\alpha \beta y=0,
$$

which is just the hypergeometric equation in its standard form, with solution

$$
y=c_{1} F(\alpha, \beta ; \gamma ; z)+c_{2} z^{1-\gamma} F(\alpha-\gamma+1, \beta-\gamma+1 ; 2-\gamma ; z) .
$$

\section{Appendix C.1. Electric multipoles}

The coupled system (3.7)-(3.8) is equivalent to solve a second-order differential equation for $K$ of the Heun type. The procedure is as follows. First, solve algebraically Eq. (3.8) for $\tilde{H}_{1}$ and substitute it into Eq. (3.7), thus obtaining a second-order differential equation for $K$. Introduce then the new variable $z=x^{2}$ togheter with the rescaling

$$
K(z)=f(z) y(z), \quad f(z)=z^{\alpha_{1}}(z-1)^{\alpha_{2}}(z-a)^{\alpha_{3}}, \quad a=-\frac{L}{2} \frac{(L-2)}{\left(L+2 \Omega^{2}\right)} .
$$

The function $y$ thus satisfies an equation of the form (C.1) with parameters

$$
\begin{aligned}
& \gamma=2 \alpha_{1}+\frac{3}{2}, \quad \delta=2 \alpha_{2}+1, \quad \epsilon=2 \alpha_{3}-1, \\
& \alpha=\alpha_{1}+\alpha_{2}+\alpha_{3}+\frac{1}{4}-\frac{\sigma_{3}}{4}, \quad \beta=\alpha+\frac{\sigma_{3}}{2}, \\
& q=3\left(\alpha_{1}+\alpha_{2}+1\right)(1-a)+a(\alpha \beta-2)-3 \alpha_{2}+\frac{L}{4},
\end{aligned}
$$


and

$$
\alpha_{1}=-\frac{1}{4}+\frac{\sigma_{1}}{4} \sqrt{1+4 L}, \quad \alpha_{2}=-\mathrm{i} \sigma_{2} \frac{\Omega}{2}, \quad \alpha_{3}=2,
$$

with $\sqrt{1+4 L}=2 l+1$ and $\sigma_{1}, \sigma_{2}, \sigma_{3}$ taking independently the values \pm 1 . The signs have to be chosen so as to avoid singular behaviours at $z=0$ and $z=1$.

\section{Appendix C.2. Magnetic multipoles}

The same procedure outlined above for the case of electric multipoles can be followed here to reduce the coupled system (3.22) -(3.23) to a second-order differential equation for $h_{1}$ of the Heun type. First, eliminate $\tilde{h}_{0}$ in favour of $h_{1}$, then introduce the new variable $z=x^{2}$ and a rescaling $h_{1}(z)=f(z) y(z)$ of the form (C.4). The function $y$ thus satisfies an equation of the form (C.1) with parameters

$$
\begin{aligned}
& \gamma=2 \alpha_{1}-\frac{1}{2}, \quad \delta=2 \alpha_{2}+3, \quad \epsilon=2 \alpha_{3}, \\
& \alpha=\alpha_{1}+\alpha_{2}+\frac{3}{4}-\frac{\sigma_{3}}{4}, \quad \beta=\alpha+\frac{\sigma_{3}}{2}, \\
& q=\alpha \beta a,
\end{aligned}
$$

and

$$
\alpha_{1}=\frac{3}{4}+\frac{\sigma_{1}}{4} \sqrt{1+4 L}, \quad \alpha_{2}=-1-\mathrm{i} \sigma_{2} \frac{\Omega}{2}, \quad \alpha_{3}=0,
$$

implying that $\epsilon=0$. Therefore in this case the Heun equation reduces to the hypergeometric equation (C.2).

\section{Appendix D. The Kovacic algorithm: an overview}

The Kovacic algorithm applies to a second-order ordinary differential equation of normal form

$$
\frac{\mathrm{d}^{2} y}{\mathrm{~d} r^{2}}=W(r) y,
$$

when $W(r)$ is a given element of $\mathbb{C}(r)$, the field of rational functions with coefficients in the field of complex numbers. Kovacic proved that all Liouvillian solutions to Eq. (D.1) belong to three mutually exclusive types. Furthermore, for each type, he provided an algorithm which determines whether a Liouvillian solution exists for that type, and constructs the solution in case it does exist. The types are as follows:

Type 1. The differential equation has a solution of the form $\mathrm{e}^{\int \tilde{\Omega} \mathrm{d} r}$, where $\tilde{\Omega} \in \mathbb{C}(r)$.

Type 2. The differential equation has a solution of the form $\mathrm{e}^{\int \tilde{\Omega} \mathrm{d} r}$, where $\tilde{\Omega}$ is algebraic over $\mathbb{C}(r)$ of degree 2 (i.e., $\tilde{\Omega}$ is a solution of a polynomial equation of degree 2 with coefficients in $\mathbb{C}(r)$ ), and solutions of type 1 form an empty set.

Type 3. All solutions of the differential equation are algebraic over $\mathbb{C}(r)$ of degree 2 , and solutions of type 1 and 2 form two empty sets.

If none of the previous cases applies, the differential equation has no Liouvillian solution. The construction of each type of Liouvillian solution is derived from the pole structure of the rational function. Kovacic also discussed some conditions that are necessary for cases 1, 2 or 3 to hold. For type 1 every pole of $W(r)$ should have even order or else order 1 , and the pole at $\infty$ should have either even order or order 
greater than 2 . For type 2 a necessary condition is that $W(r)$ must have at least one pole that has either odd order greater than 2 or order 2 . Last, for type 3 the order of a pole of $W(r)$ cannot exceed 2 , and the order of the pole at $\infty$ must be at least 2 .

Let us discuss in detail the case 1 . The remaining cases 2 and 3 apply as well and can be treated similarly.

For a fixed rational function $W(r)$, type 1 Liouvillian solutions of Eq. (D.1) are determined in the following way. A finite set of constants $d$ is derived algorithmically from the pole structure of $W(r)$ and for each $d$ a function $\tilde{\Omega}(r)$ is also constructed. If any $d$ is a non-negative integer $n$ and, for the corresponding $\tilde{\Omega}(r)$, the differential equation

$$
\frac{\mathrm{d}^{2} \mathcal{P}(r)}{\mathrm{d} r^{2}}+2 \tilde{\Omega} \frac{\mathrm{d} \mathcal{P}(r)}{\mathrm{d} r}+\left[\frac{\mathrm{d} \tilde{\Omega}}{\mathrm{d} r}+\tilde{\Omega}^{2}-W(r)\right] \mathcal{P}(r)=0
$$

has a polynomial solution $\mathcal{P}$ of degree $n$, then Eq. (D.1) has a Liouvillian solution given by

$$
y=\mathcal{P} \mathrm{e}^{\int \tilde{\Omega} \mathrm{d} r} .
$$

If this is not the case for any $d$, then Eq. (D.1) has no type 1 solutions.

Since $W(r)$ depends on different independent parameters, several different pole structures may occur by imposing conditions on the parameters which alter the order of poles. For each different pole structure, in general, $d$ and $\tilde{\Omega}$ are expressed in terms of the parameters. Solutions to Eq. (D.1) may be obtained by requiring $d=n$, which then is just a constraint on the parameters, and finding all sets of parameters for which Eq. (D.2) has a polynomial solution of degree $n$.

\section{Acknowledgement}

ICRANet is thanked for support and Dr. V. Montaquila for stimulating discussion. GE is grateful to Dipartimento di Scienze Fisiche of Federico II University, Naples, for hospitality and support, and he dedicates to Maria Gabriella his contribution to this work.

\section{References}

[1] Guth A H 1981 Phys. Rev. D 23347

[2] Bass S D 2011 J. Phys. G: Nucl. Part. Phys. 38043201

[3] Gibbons G W and Hawking S W 1977 Phys. Rev. D 152738

[4] Lohiya D and Panchapakesan N 1978 J. Phys. A: Math. Gen. 111963

[5] Lohiya D and Panchapakesan N 1979 J. Phys. A: Math. Gen. 12533

[6] Biswas S, Guha J and Sarkar N G 1995 Class. Quantum Grav. 121591

[7] Suzuki H and Takasugi E 1996 Mod. Phys. Lett. A 11431

[8] Hawking S W and Ellis G F R 1973 The Large Scale Structure of Spacetime (Cambridge, UK: Cambridge University Press)

[9] Regge T and Wheeler J A 1957 Phys. Rev. 1081063

[10] Teukolsky S 1973 Ap. J. 185635

[11] Guven J and Núñez D 1990 Phys. Rev. D 422577

[12] Khanal U and Panchapakesan N 1981 Phys. Rev. D 24829

[13] Newman E T and Penrose R 1962 J. Math. Phys. 3566

[14] Jackson J 1975 Classical Electrodynamics (New York: Wiley)

[15] Chandrasekhar S 1983 The Mathematical Theory of Black Holes (Oxford: Clarendon Press)

[16] Gal'tsov D V and Núñez D 1989 Gen. Rel. Grav. 21257

[17] Kovacic J J 1986 J. Symb. Comput. 23 
[18] Bini D, Capozziello S and Esposito G 2008 Int. J. Geom. Meth. Mod. Phys. 5 1069; erratum 2009 ibidem 6367

[19] Ferrari V, Pendenza P and Veneziano G 1988 Gen. Rel. Grav. 201185

[20] Heun K 1889 Math. Ann. 33161

[21] Ronveaux A 1995 Heun's Differential Equations (Oxford: Oxford University Press) 